\title{
Cholesterol Perturbs Lipid Bilayers Nonuniversally
}

\author{
Jianjun Pan, Thalia T. Mills, Stephanie Tristram-Nagle, and John F. Nagle \\ Physics Department, Carnegie Mellon University, Pittsburgh, Pennsylvania 15213, USA
}

(Received 22 February 2008; published 15 May 2008)

\begin{abstract}
Cholesterol is well known to modulate the physical properties of biomembranes. Using modern x-ray scattering methods, we have studied the effects of cholesterol on the bending modulus $K_{C}$, the thickness $D_{H H}$, and the orientational order parameter $S_{\text {xray }}$ of lipid bilayers. We find that the effects are different for at least three classes of phospholipids characterized by different numbers of saturated hydrocarbon chains. Most strikingly, cholesterol strongly increases $K_{C}$ when both chains of the phospholipid are fully saturated but not at all when there are two monounsaturated chains.
\end{abstract}

DOI: 10.1103/PhysRevLett.100.198103

PACS numbers: 87.16.dm, 87.14.Cc, 87.64.Bx

Despite the popular perception that cholesterol is unhealthy, it is a vital component in the membranes of mammalian cells, with concentrations varying up to $50 \mathrm{~mol} \%$ of the total lipid in healthy cell plasma membranes [1]. Cholesterol is considered to be a key molecule for the formation of small rafts that have been implicated in providing favorable nano environments to facilitate biochemical functioning of membrane proteins [2]. Cholesterol is important in producing macroscopic fluidfluid phase separation that has been so strikingly visualized by fluorescence microscopy [3]. Not surprisingly, there is a large biophysical literature of cholesterol in lipid bilayers $[4,5]$.

Cholesterol has a short, thermally flexible, hydrocarbon tail attached to a larger, rigid, hydrophobic, fused ring structure headed with a small, hydrophilic, hydroxyl group that is thought to be sufficient to anchor the ring end of cholesterol to the aqueous interface. In contrast, lipid molecules have a larger hydrophilic headgroup and longer, flexible, hydrocarbon tails. Many studies have shown that addition of cholesterol increases the orientational order parameter $S_{\mathrm{mol}}$ of the hydrocarbon tails of the lipids, thereby partially straightening the chains and preferentially orienting them along the bilayer normal, perpendicular to the membrane [5]. Both straightening and orientational ordering promote the observed thickening of the hydrocarbon portion of the bilayer. Other studies have shown that addition of cholesterol makes bilayers less flexible in two ways: first, by increasing the bending modulus $K_{C}$ and thereby increasing the energy $(1 / 2) K_{C} C^{2}$ of curvature $C$, and second, by increasing the compression modulus $K_{A}$ and the energy $(1 / 2) K_{A}\left(A-A_{0}\right)^{2}$ required to compress or dilate the area from its free energy minimum at $A_{0}$ [6]. Many of these studies have focused on phosphatidylcholine (PC) lipids, like DMPC or DPPC, that have both their hydrocarbon chains fully saturated $(\mathrm{C}-\mathrm{C}$ single bonds between all the carbons), or the studies have focused on lipids, like SOPC or POPC, that have one saturated chain and one monounsaturated chain (one $\mathrm{C}=\mathrm{C}$ double bond about halfway along the otherwise saturated hydrocarbon chain). Natural biomembranes have lipids with different numbers of saturated chains. A recent study of just one lipid, POPC, with three similar sterols, cholesterol, lanosterol, and ergosterol, has suggested that there is universal behavior involving the hydrocarbon thickness $d, S_{\text {mol }}$ (both obtained via the first NMR moment $M_{1}$ ), $K_{A}$, and $K_{C}$ [5]. In this Letter we report that the effect of cholesterol is not obviously universal when it is added to different lipids with different numbers of saturated chains.

Our experiments were performed on samples consisting of stacks of approximately 2000 well oriented bilayers deposited on smooth, flat silicon wafers [7]. These samples were brought to within a few Ångstroms of full hydration so the water space between bilayers was sufficiently large (ca. 15-20 $\AA$ ) that the interbilayer interaction is a negligible perturbation compared to the intrabilayer interactions responsible for the properties of individual bilayers [8]. These fully hydrated, fluid phase, smectic liquid crystalline, soft matter samples undergo considerable spontaneous thermal fluctuations. Such fluctuations degrade x-ray diffraction peaks [9], which has made traditional structural analysis difficult. However, these smectic fluctuations produce considerable diffuse $\mathrm{x}$-ray scattering that can easily be measured on a CCD using synchrotron sources as shown in Fig. 1. The diffuse scattering is continuous in $q$ space and provides more information than can be obtained from the conventional diffraction peaks when an analysis employing liquid crystal theory is carried out [10].

The first part of the analysis of the diffuse scattering intensity $I(q)$ obtains the bending modulus $K_{C}$, as well as the compression modulus $B$; the latter is of interest for interactions between bilayers that will not be discussed here. From these two moduli and the smectic liquid crystal theory, the interbilayer structure (interference) factor $S(q)$ is evaluated and then divided into the measured intensity $I(q)=S(q)|F(q)|^{2} / q$ to obtain the intrabilayer "form" factor $|F(q)|$ from which the second part of the analysis obtains the electron density profile $\rho(z)$ along the $z$ direction perpendicular to the membrane [7]. The thickness of the membrane in this Letter is the distance between the two 


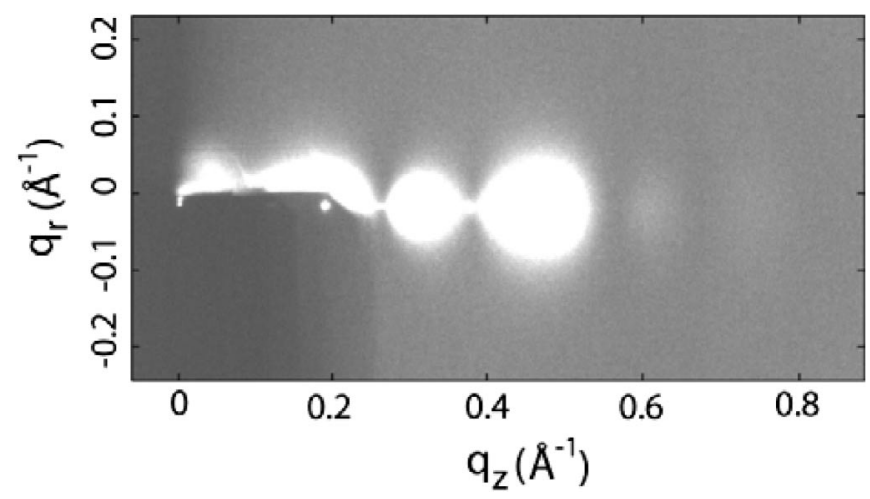

FIG. 1. CCD image of low angle diffuse scattering of DOPC with $30 \%$ cholesterol at $30{ }^{\circ} \mathrm{C}$. The dark region $q_{z}<0.2, q_{r}<$ 0.01 is blocked by a semitransparent beam stop that attenuates the beam and the very strong first two lamellar peaks. $K_{C}$ is related to the $q_{r}$ width and $D_{H H}$ to the $q_{z}$ distribution of intensity $[7,10]$.

maxima in the electron density profile; this is often called the head-head thickness $D_{H H}$ because the maxima in $\rho(z)$ essentially locate the electron dense phosphate in the lipid headgroups that reside in the two interfacial regions that define the thickness of the bilayer.

The third physical parameter that we measure is essentially the average orientational order parameter of the hydrocarbon chains. This fundamental quantity is often called $S_{\text {mol }}$ and is usually measured using NMR techniques. However, the underlying information is also embedded in the so-called wide angle x-ray scattering shown in Fig. 2. Analysis of these data obtains a molecular order parameter that is named $S_{\text {xray }}$ [11]. Because obtaining a consistent measure of molecular orientation using NMR is

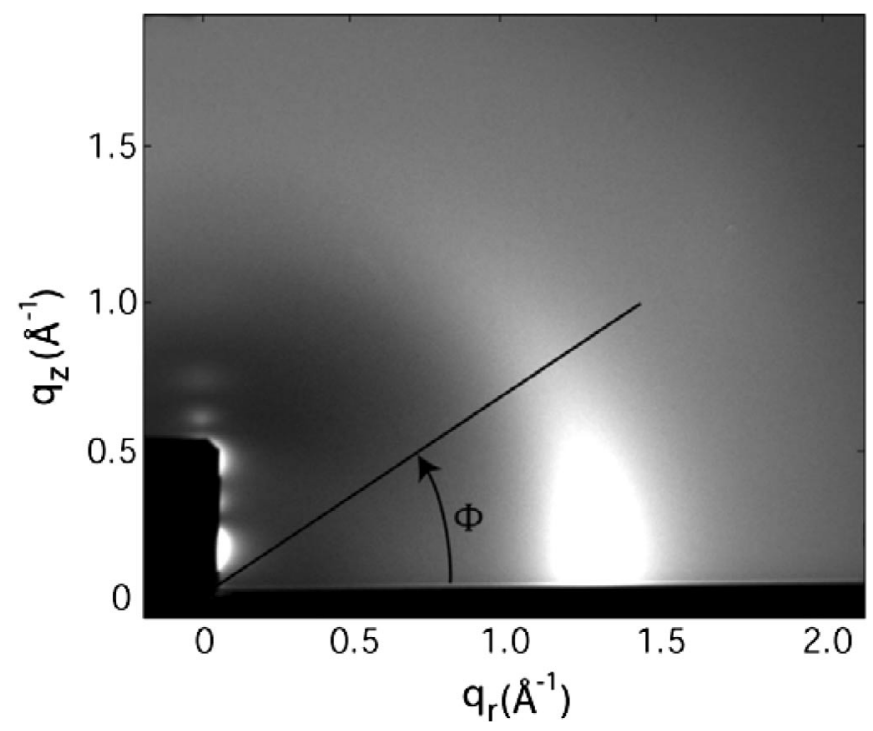

FIG. 2. CCD image of wide angle scattering of DOPC with $30 \%$ cholesterol at $30{ }^{\circ} \mathrm{C}$. The beam stop blocks the first five lamellar orders at $q_{r}=0 . S_{\text {xray }}$ decreases as the width of the intensity distribution increases in the $\phi$ direction [11]. problematic for unsaturated chains, we suggest that $S_{\text {xray }}$ is an appropriate substitute. Indeed, $S_{\text {xray }}$ tracks $S_{\mathrm{NMR}}$ quite well as cholesterol is added to saturated DPPC, differing only by a normalization factor [11].

Figure 3 shows results for our three physical parameters as a function of cholesterol mole fraction $c$ for several lipids. Our most dramatic result is for the bending modulus $K_{C}$ shown in Fig. 3(a). While $K_{C}$ for saturated DMPC increases more than fourfold with $30 \%$ cholesterol, for SOPC with one monounsaturated chain it increases only about twofold and, most surprisingly, $K_{C}$ remains essentially constant for two lipids that have both their chains monounsaturated, DOPC (18 carbons/chain) and diC22:1 (22 carbons/chain). We suggest a simple, working, phenomenological description in this Letter, namely, that the effect of cholesterol on $K_{C}$ increases with the number of saturated chains in the lipid.

As expected from the literature, we confirm that cholesterol generally induces greater orientational order [Fig. 3(b)] and it also thickens these bilayers [Fig. 3(c)].

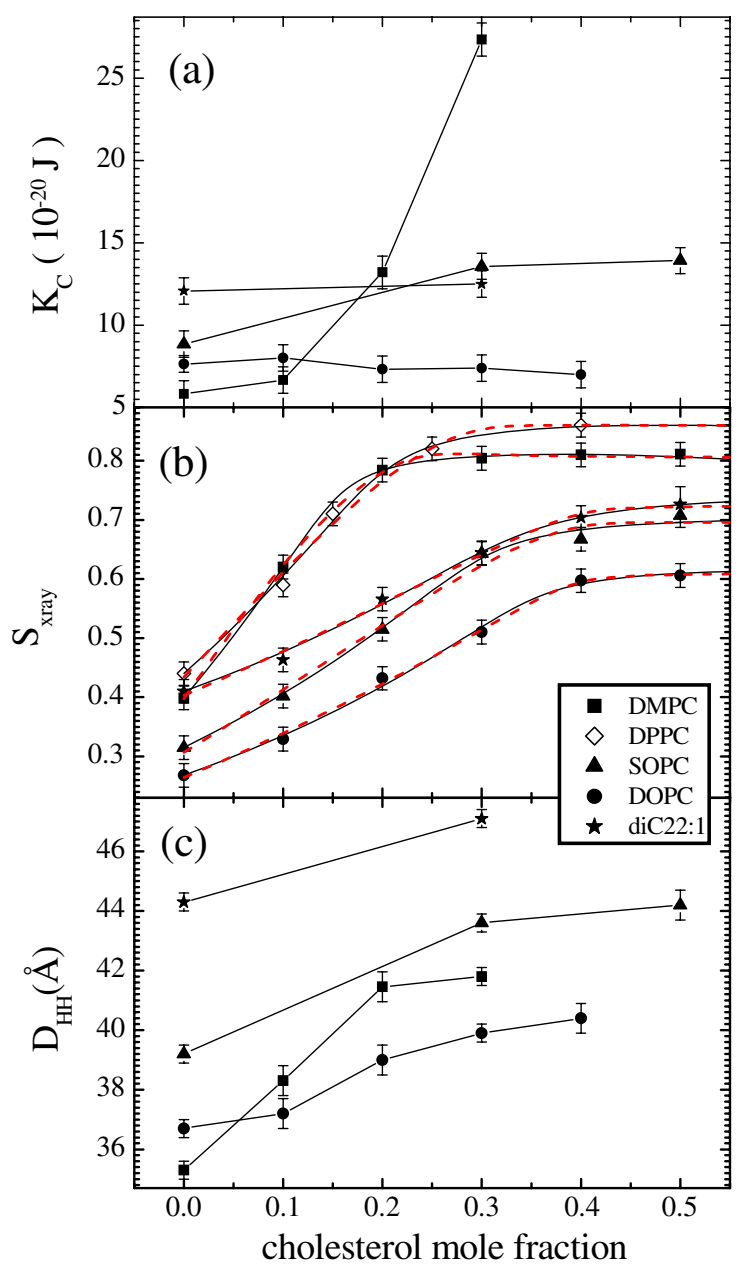

FIG. 3 (color online). (a) Bending modulus, (b) orientational order parameter, and (c) thickness as a function of cholesterol mole fraction $c$. The solid curves in (b) were fits to Eq. (1) and the dashed curves were fits to Eq. (2) with values of the parameters in Table I. 
Of greater current interest is that a progression with the number of saturated chains, similar though less dramatic than the one for $K_{C}$, can also be discerned from our results for $S_{\text {xray }}$ in Fig. 3(b). The initial slopes for $S_{\text {xray }}$ at low cholesterol concentration are greater for DMPC (14 carbons/chain) and DPPC (16 carbons/chain) that have two saturated chains than for DOPC and diC22:1 that have two monounsaturated chains, and the initial slope is intermediate for SOPC with one saturated chain and one monounsaturated chain. For the fully saturated lipids there is a clear leveling off for $c \sim 0.2$. A leveling off for the unsaturated lipids is suggested, but would occur only at higher values of $c$ that are experimentally difficult due to cholesterol crystallization [12,13]. Our results for $D_{H H}$ in Fig. 3(c) also are consistent with the same progression as for $S_{\text {xray }}$. A similar progression for $D_{H H}$ has recently been reported for DMPC, SOPC, and DOPC [13].

Figure 4 searches for correlations between our three physical quantities. Figure 4(a) indicates that $D_{H H}$ and $S_{\text {xray }}$ are well correlated for each lipid. Of course, lipids with longer chains will have larger $D_{H H}$ even if the $S_{\text {xray }}$ order parameter is the same and if there are no other differences. Beyond this obvious difference, Fig. 4(a) suggests that there might be a progression to greater slopes with more saturation. The more dramatic breakdown of universality for different lipid types is indicated in Fig. 4(b). For DOPC and diC22:1 with no saturated chains, $K_{C}$ is essentially constant as $S_{\text {xray }}$ increases with added cholesterol, whereas $K_{C}$ increases for SOPC with one saturated chain, and it increases dramatically for DMPC with both chains saturated.

Another universal relation often cited [14] is $K_{C}=$ $K_{A} d^{2} / n$, where $d$ is usually thought of as the hydrocarbon thickness, $d \sim D_{H H}-10 \AA$ [7]. This relation is well satisfied for single component lipids when $n=24$, as predicted by the polymer brush model [14]. However, it has recently been reported that it breaks down when cholesterol is added to the singly monounsaturated POPC lipid [5]. Although our data cannot provide a direct test because we do not measure $K_{A}$, our results for $d$ and $K_{C}$ for DOPC would, by this relation, require $K_{A}$ to decrease by $\sim 25 \%$ upon addition of $30 \%$ cholesterol, and such a decrease is inconsistent with a recent report that $K_{A}$ increases by $\sim 50 \%$ upon addition of $30 \%$ cholesterol to DOPC [15]. Also, our $K_{C}$ and $d$ results for SOPC would predict that $K_{A}$ should increase by $13 \%$ when $30 \%$ cholesterol is added, but the increase has been reported to be closer to $100 \%$ [15].

Because our most egregious breakdown of universality involves $K_{C}$, it is appropriate to ask if our measurement technique is reliable. For single component lipid bilayers our results are in good agreement with those obtained using the aspiration pipette method [14]. Although the remarkable progression with amount of saturation for the effect of cholesterol on $K_{C}$ has not been pointed out in the literature, there have been some reports consistent with our results.

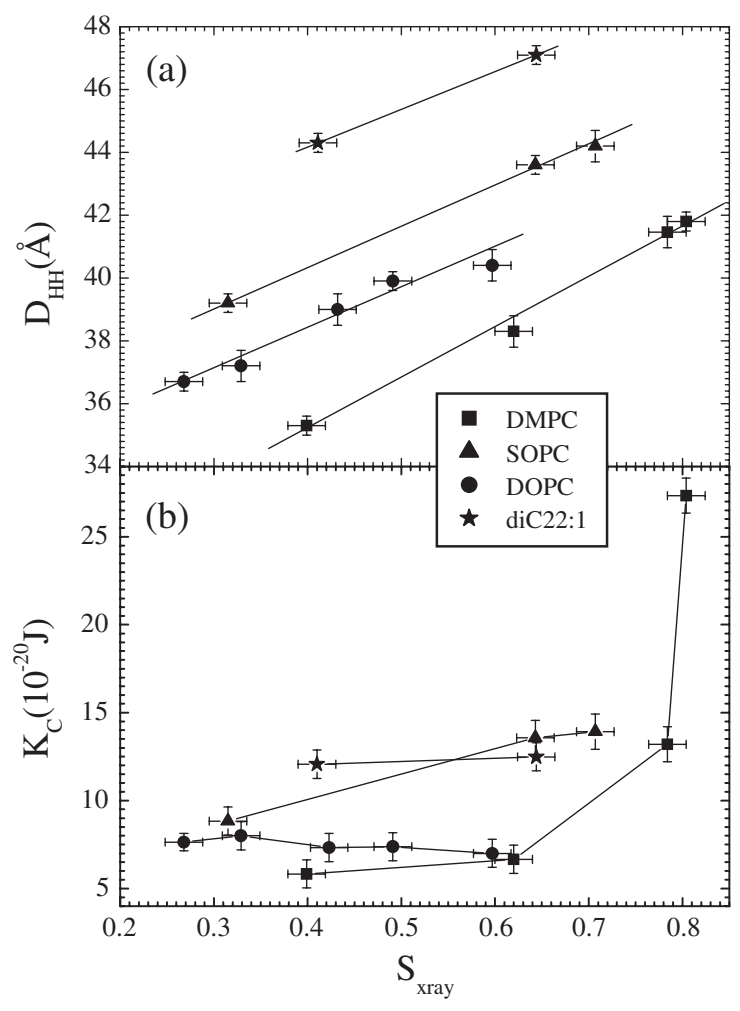

FIG. 4. (a) Thickness $D_{H H}$ and (b) bending modulus $K_{C}$ versus orientational order parameter $S_{\text {xray }}$.

For DMPC with $30 \%$ cholesterol at $30{ }^{\circ} \mathrm{C}$ the bending modulus increases by a factor of 3.5 [16] or 3.2 [17] by analysis of fluctuations in giant unilamellar vesicles. While a factor of 2.7 for SOPC [6] was almost as large as for DMPC, that was for a larger cholesterol concentration of $50 \%$. For POPC at $30 \%$ cholesterol a factor of 2.3 has been reported [5]. In contrast, for DOPC a method involving hexagonal phases with tetradecane additive indicated no change in $K_{C}$ up to $30 \%$ cholesterol followed by a factor of only 1.3 up to $50 \%$ cholesterol [18]. A recent analysis of $\mathrm{x}$ ray scattering from unoriented samples found that the Caillé $\eta$ parameter [9], which is inversely proportional to the square root of the product of $K_{C}$ and $B$, decreased more for DMPC than for POPC upon addition of cholesterol [19].

The theory of lipid bilayers with cholesterol is challenging due to the complex molecular interactions. Coarse grained simulation models have been studied in which each molecule has only translational degrees of freedom with phenomenological pairwise interactions or with multibody interactions to account for the possibility that the headgroups of the lipids could provide an "umbrella" to shield the cholesterol from direct contact with water [20]. Other theories postulate that cholesterol creates a condensed complex consisting of one cholesterol and $p$ lipids $[13,21]$. The simplest such theory [21] employs a simple chemical reaction model, free cholesterol $+p$ free lipids $\Leftrightarrow$ complex with an equilibrium constant $K_{\text {eq }}=x /(c-$ $x)(1-c-p x)^{p}$, where $x$ is the mole fraction of com- 
TABLE I. Values of parameters from fits to Eqs. (1) and (2). $N$ is the number of fully saturated chains.

\begin{tabular}{lcccc}
\hline \hline & & Equation (1) & \multicolumn{2}{c}{ Equation (2) } \\
& $N$ & $p$ & $p$ & $\gamma$ \\
\hline DMPC & 2 & 3.9 & 2.5 & 1.5 \\
DPPC & 2 & 2.9 & 2.0 & 1.0 \\
SOPC & 1 & 2.0 & 1.5 & 0.5 \\
DOPC & 0 & 1.7 & 1.5 & 0.2 \\
diC22:1 & 0 & 1.7 & 1.5 & 0.2 \\
\hline \hline
\end{tabular}

plexed cholesterol and $c$ is the total cholesterol mole fraction. In Fig. 3(b) we have fit our data to

$$
S_{\text {xray }}=S_{F} f_{F}+S_{C} f_{C}
$$

where $f_{C}=p x /(1-c)$ is the fraction of complexed lipid with order parameter $S_{C}$ and $f_{F}=1-f_{C}$ is the fraction of uncomplexed lipid with order parameter $S_{F}$. The values of $p$ from this fit are given in Table I.

The chemical reaction model is clearly oversimplified for describing a condensed matter phase, so we have also combined it with a different theory that is more realistic in that it allows the cholesterol complexes to perturb the properties of uncomplexed lipids [13]. While the perturbation on the uncomplexed lipids should decay with a correlation length $\xi$, the theory accounts for the increase in the average order parameter of the uncomplexed lipid fraction $f_{F}$ due to the influence of the fraction of clusters $f_{C} / p$ in a mean field, phenomenological way by writing

$$
S_{\text {xray }}=\left(S_{F}+\gamma f_{C} / p\right) f_{F}+S_{C} f_{C}
$$

where $\gamma$ parametrizes the strength of the coupling. Although in [13] it was assumed that $K_{\text {eq }}$ was infinite, in Fig. 3(b) we show the fit to our $S_{\text {xray }}$ data using a finite value of $K_{\text {eq }}$ and the values of $p$ and $\gamma$ are given in Table I.

Both models obtain larger values of $p$ for saturated lipids. This is required in order that the curves in Fig. 3(b) level off in the experimental range, whereas the curves with smaller $p$ for the unsaturated lipids would level off at larger cholesterol concentration. Our values of $\gamma$ increase with the number of saturated chains [22]. This is consistent with saturated chains being more strongly correlated and/or having a longer correlation length $\xi$. Although we allowed $K_{\text {eq }}$ to be a free parameter in both models, it is an insensitive parameter and the fits differed very little compared to fixing $K_{\text {eq }}$ to the value 1270 given in [21].

In conclusion, our $S_{\text {xray }}$ results imply that cholesterol has a larger effect on lipids that have more saturated chains in two ways: (1) There are more proximal lipids that may be considered bound in a complex (larger $p$ ), and (2) the chains in the distal lipids are more susceptible to ordering by cholesterol perturbation (larger $\gamma$ ). These trends in our $S_{\text {xray }}$ results are consistent with the even more dramatic trend with the number of saturated chains that we observe for the effect of cholesterol on $K_{c}$. If a universal description exists for the physical effects of cholesterol on biomembranes, it would require, at least, the inclusion of the particular molecular structure of the lipids, such as the number of saturated chains studied here, and probably also other natural lipid variations, such as having more than one double bond and having different headgroups.

This research was supported by NIH Grant No. GM 44976. Synchrotron beam time was provided by CHESS, which is funded by NSF Grant No. DMR0225180.

[1] P. Emmelot, in Mammalian Cell Membranes, edited by G. A. Jamieson and D. M. Robinson (Butterworths, London, 1977), Chap. 1, Vol. 2.

[2] Biophysical Society Discussions 2004. Probing Membrane Microdomains. http://www.biophysics.org/discussions/ 2004/.

[3] T. Baumgart, S. T. Hess, and W.W. Webb, Nature (London) 425, 821 (2003).

[4] Cholesterol in Membrane Models, edited by L.X. Finegold (CRC Press, Boca Raton, 1993).

[5] J. Henriksen et al., Biophys. J. 90, 1639 (2006).

[6] E. Evans and W. Rawicz, Phys. Rev. Lett. 64, 2094 (1990).

[7] N. Kučerka et al., Biophys. J. 88, 2626 (2005).

[8] J. F. Nagle, Annu. Rev. Phys. Chem. 31, 157 (1980).

[9] A. Caillé, C.R. Acad. Sci., Ser. B 274, 891 (1972).

[10] Y. Lyatskaya et al., Phys. Rev. E 63, 011907 (2000).

[11] T. T. Mills et al., Biophys. J. BioFAST: April 4, 2008. doi: 10.1529/biophysj.107.127910.

[12] J. Y. Huang, J. T. Buboltz, and G. W. Feigenson, Biochim. Biophys. Acta 1417, 89 (1999).

[13] W. C. Hung et al., Biophys. J. 92, 3960 (2007).

[14] W. Rawicz et al., Biophys. J. 79, 328 (2000); We note that J. Pan et al., Biophys. J. 94, 117 (2008), have recently found that the polymer brush model is outstanding in predicting additional properties of single component bilayers.

[15] W. Rawicz et al., Biophys. J. BioFAST: March 13, 2008. doi: 10.1529/biophysj.107.121731.

[16] H. P. Duwe, J. Kaes, and E. Sackmann, J. Phys. (Paris) 51, 945 (1990).

[17] P. Méléard et al., Biophys. J. 72, 2616 (1997).

[18] Z. Chen and R. P. Rand, Biophys. J. 73, 267 (1997).

[19] A. Hodzic et al., Biophys. J. BioFAST: January 30, 2008. doi: 10.1529/biophysj.107.123224.

[20] J. Y. Huang and G. W. Feigenson, Biophys. J. 76, 2142 (1999).

[21] H. McConnell and A. Radhakrishnan, Proc. Natl. Acad. Sci. U.S.A. 103, 1184 (2006).

[22] The trend with saturation that we see for $\gamma$ in our $S_{\text {xray }}$ data is the same as reported for $D_{H H}$ in [13]. However, the data obtained in [13] were cut off at high $c$ by cholesterol crystallization, which led to an interpretation that did not have $p$ depend significantly on chain saturation. 